\title{
Evolution and maintenance of microbe-mediated protection under occasional pathogen infection
}

\author{
Anke Kloock (D) | Michael B. Bonsall (D) | Kayla C. King (D)
}

Department of Zoology, University of Oxford, Oxford, UK

\section{Correspondence}

Anke Kloock, Department of Zoology, University of Oxford, 11a Mansfield Road, Oxford OX1 3SZ, UK.

Email: anke.kloock@zoo.ox.ac.uk

\section{Funding information}

FP7 Ideas: European Research Council, Grant/Award Number: COEVOPRO802242; Leverhulme Trust, Grant/Award Number: RPG-2015-165; Studienstiftung des Deutschen Volkes

\begin{abstract}
Every host is colonized by a variety of microbes, some of which can protect their hosts from pathogen infection. However, pathogen presence naturally varies over time in nature, such as in the case of seasonal epidemics. We experimentally coevolved populations of Caenorhabditis elegans worm hosts with bacteria possessing protective traits (Enterococcus faecalis), in treatments varying the infection frequency with pathogenic Staphylococcus aureus every host generation, alternating host generations, every fifth host generation, or never. We additionally investigated the effect of initial pathogen presence at the formation of the defensive symbiosis. Our results show that enhanced microbe-mediated protection evolved during host-protective microbe coevolution when faced with rare infections by a pathogen. Initial pathogen presence had no effect on the evolutionary outcome of microbe-mediated protection. We also found that protection was only effective at preventing mortality during the time of pathogen infection. Overall, our results suggest that resident microbes can be a form of transgenerational immunity against rare pathogen infection.
\end{abstract}

\section{KEYWORDS}

defensive symbiosis, experimental evolution, heterogeneity, host-pathogen interactions, protection

\section{1 | INTRODUCTION}

In nature, all plants and animals are colonized by microbes (Barrière, \& Felíix, 2005; Ley, Peterson, \& Gordon, 2006; Vántus, Kovács, \& Zsolnai, 2014). The composition of these microbial communities is highly diverse and includes harmful, neutral, and beneficial microbial species (Ley et al., 2006), including those that can be important players in host defense against parasites, a phenomenon referred to as "defensive mutualism" (King, 2019; May \& Nelson, 2014). Recognized for over a century, defensive mutualism has been observed in plants (Mendes et al., 2011) and in a range of animals (Dillon, Vennard, \& Charnley, 2000; Dong, Manfredini, \& Dimopoulos, 2009; Jaenike, Unckless, Cockburn, Boelio, \& Perlman, 2010; Koch \&
Schmid-Hempel, 2011), including humans (Kamada, Seo, Chen, \& Núñez, 2013; Ley et al., 2006; Maynard, Elson, Hatton, \& Weaver, 2012) wherein microbes can supplement host immune systems (Abt \& Artis, 2013; Hooper, Littman, \& Macpherson, 2012; McFall-Ngai et al., 2013).

The net benefits of defensive mutualism are dependent upon the presence of pathogens (Clay, Holah, \& Rudgers, 2005; King \& Bonsall, 2017; Lively, Clay, Wade, \& Fuqua, 2005). While hosts can benefit from microbe-mediated protection, defensive symbionts can be less beneficial to the host in the absence of enemies, due to metabolic and physiological costs (King, 2019). For example, in the interaction of aphids and the bacterium Hamiltonella defensa, the host tissue is harmed by defensive toxins that protect against 
infection from parasitoids (Vorburger \& Gouskov, 2011). In some cases, possessing protective microbes might be more beneficial to the host than investing in its own immune system (Martinez et al., 2016). From the perspective of the symbiont, it is most useful to its host under high pathogen prevalence and thus can persist in the host population (Palmer et al., 2008). Nevertheless, a stable symbiotic interaction is hypothesized to be evolved and maintained (Kwiatkowski \& Vorburger, 2012) only when the host benefit of carrying defensive symbionts outweighs any costs. The interactions of obligate and defensive symbionts and hosts can be stable for millions of years (Moran, Tran, \& Gerardo, 2005).

Not all environments are constantly pathogen-rich, which might shift the balance of costs and benefits during defensive mutualisms, particularly during coevolutionary interactions (King \& Bonsall, 2017). Pathogen prevalence can be spatially (King, Delph, Jokela, \& Lively, 2009) or temporally variable, the latter in the case of seasonal epidemics (e.g., flu peaks each winter in the Northern Hemisphere (Finkelman, 2007) or rabies in North American skunks, which peaks in Autumn (Gremillion-Smith \& Woolf, 1988). Different environmental factors can influence disease transmission such as an increase in malaria risk in warmer regions after rainfall (Altizer, Dobson, Hosseini, Hudson, \& Pascual, 2006) or an increase in contact rate and thus higher flu infection rate during the winter months (London \& Yorke, 1973). The impact of other temporally heterogeneous factors on the strength and direction of selection on species interactions have been explored (oxygen concentration [Dey, Proulx, \& Teotónio, 2016], resource availability [Friman \& Laakso, 2011; Friman, Laakso, Koivu-Orava, \& Hiltunen, 2011; Hiltunen, Friman, Kaitala, Mappes, \& Laakso, 2012], environmental productivity [Harrison, Laine, Hietala, \& Brockhurst, 2013]). Whether the varied presence of pathogens can similarly alter selection for symbiotic interactions has been explored theoretically (Fenton, Johnson, Brownlie, \& Hurst, 2011), but remains to be empirically tested.

Here, we examined the impact of temporal variation in pathogen infection on the evolution of microbe-mediated protection. We used Caenorhabditis elegans as a worm host and allowed it to be colonized by a bacterium (Enterococcus faecalis) that protects against infection by Staphylococcus aureus (King et al., 2016). Enterococcus faecalis has been shown to be protective across animal microbiomes (Kommineni et al., 2015; Martín-Vivaldi et al., 2010). It has been previously shown that $E$. faecalis can evolve to provide enhanced protection when residing in $C$. elegans hosts during constant pathogen infection (King et al., 2016; Rafaluk-Mohr, Ashby, Dahan, \& King, 2018). From this, we predict that variation in pathogen infection might limit the evolution of microbe-mediated protection. In the present study, we experimentally copassaged $C$. elegans with protective $E$. faecalis and infected the host with evolutionary static pathogenic $S$. aureus at different intervals of host evolution. We also examined whether pathogen presence at the initial formation of the coevolving interaction is crucial to the evolution of protection. We show that enhanced microbe-mediated protection emerged out of novel coevolutionary host-microbe interactions and during pathogen infection, regardless of its temporal variability or the time point of first infection.
Enhanced protection was only effective during pathogen infection. If hosts survived infection, they could recover and had the same longevity and reproductive output across treatments. These results thus suggest that even occasional pathogen infection can select for defensive mutualism, revealing the potential for this phenomenon to be widespread in nature.

\section{2 | MATERIALS AND METHODS}

\section{1 | Worm host and bacteria system}

As a bacteriovore, Caenorhabditis elegans interacts constantly with a variety of bacteria either by feeding or by hosting them (Cabreiro \& Gems, 2013; Garsin et al., 2001; Schulenburg \& Ewbank, 2004). Consequently, C. elegans is an established model for studying innate immunity (Gravato-Nobre \& Hodgkin, 2005), as it can be infected with its natural (Jansson, 1994; Schulenburg \& Ewbank, 2004) as well as opportunistic pathogens (Garsin et al., 2001; Tan, MahajanMiklos, \& Ausubel, 1999). Most pathogens are taken up orally by the worm (Marsh \& May, 2012), and some can proliferate and colonize the worm gut (King et al., 2016; Rafaluk-Mohr et al., 2018).

Naturally, C. elegans is a self-fertilizing hermaphrodite (Brenner, 1974), but in this experiment obligate outcrossing worm populations (line EEVD00) with males and females (hermaphrodites that carry the fog-2(q71) mutation) were used (Theologidis, Chelo, Goy, \& Teotónio, 2014). This lineage was generated by Henrique Teotonio (ENS Paris) and encompasses the genetic diversity of 16 natural worm isolates (Theologidis et al., 2014). Worms were kept on Nematode Growth Medium (NGM), inoculated with Salmonella, hereafter referred to as food. Worms were infected with the pathogenic S. aureus (MSSA476; Holden et al., 2004), which is virulent and kills worm hosts by lysing the intestinal cells lining the gut wall (Sifri, Begun, Ausubel, \& Calderwood, 2003). Worms were exposed to E. faecalis (OG1RF; Garsin et al., 2001), which was isolated from the human digestive system, but has been previously shown to colonize and proliferate in the host gut (Ford, Williams, Paterson, \& King, 2017; King et al., 2016; Rafaluk-Mohr et al., 2018), where it provides protection.

\section{2 | Experimental evolution-Design}

Six single clones of E. faecalis (one for each of the six replicate populations) and a single population of $C$. elegans were the ancestors (hereafter referred to as the Ancestor) for all evolving populations. To account for potential differences in virulence, a stock of four clones of S. aureus was used for pathogen infections. Both C. elegans and colonizing $E$. faecalis were allowed to evolve in the presence of each other, while $S$. aureus was kept evolutionarily static. Infection with S. aureus was varied over host evolutionary time (indicated by purple in Table 1) to represent temporal heterogeneity in pathogen infection, including a range from always to every 2 nd generation, 
TABLE 1 Experimental procedure for the evolution experiment

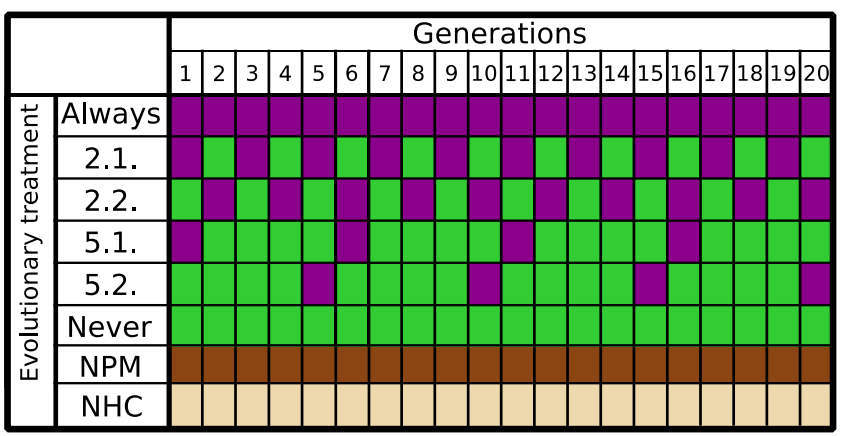

every 5th generation, and never (Table 1). Moreover, we included differences in whether pathogens were present at the initial formation of the symbiotic interaction or later (2.1. vs. 2.2., and 5.1. vs. 5.2. in Table 1). Controls for laboratory adaptation were maintained for the host (No Protective Microbe control, NPM in Table 1) and E. faecalis (No Host Control, NHC in Table 1).

Columns indicate the number of experimental host generations (1-20), while rows show the eight treatments. Host generations were infected with Staphylococcus aureus (purple) or given food (green), while constantly coevolving with Enterococcus faecalis. Two controls for laboratory effects on host evolution (dark brown, No Protective Microbe, NPM) and E. faecalis evolution (light brown, No Host Control, NHC) were also included, where the NPM treatment was only ever exposed to food alone. Each evolutionary treatment consisted of six independent evolutionary replicates.

\section{3 | Experimental evolution-Culturing and passaging methods}

At the start of each generation, worms were bleached as described previously and left in M9 buffer overnight for larvae to hatch (Stiernagle, 2006). Simultaneously, E. faecalis clones were cultured overnight in Todd-Hewitt Broth (THB) in $600 \mu \mathrm{l}$ at $30^{\circ} \mathrm{C}$, while food was cultured overnight in LB broth. Subsequently, 9-cm NGM plates were inoculated with $300 \mu \mathrm{l}$ of each overnight culture. Plates with freshly inoculated bacteria were dried at room temperature before approximately 1,000 L1 worms were added to each NGM plate. After these plates dried at room temperature, they were transferred to a $20^{\circ} \mathrm{C}$ incubator and left for $48 \mathrm{hr}$. Simultaneously, a liquid culture of $S$. aureus was grown in THB from frozen stock, while a liquid culture of food was grown in LB, and both were incubated under shaking conditions at $30^{\circ} \mathrm{C}$. The following day, $100 \mu$ l of each overnight culture was spread on 9-cm plates, S. aureus on Tryptone Soy Broth agar (TSB) plates and food on NGM plates, and incubated at $30^{\circ} \mathrm{C}$ overnight. To transfer worms to the pathogen or food plates, nematodes were washed off the $E$. faecalis plates with M9 buffer and washed three times over small-pore filters to remove all externally attached bacteria, as previously described (Jansen et al., 2015;

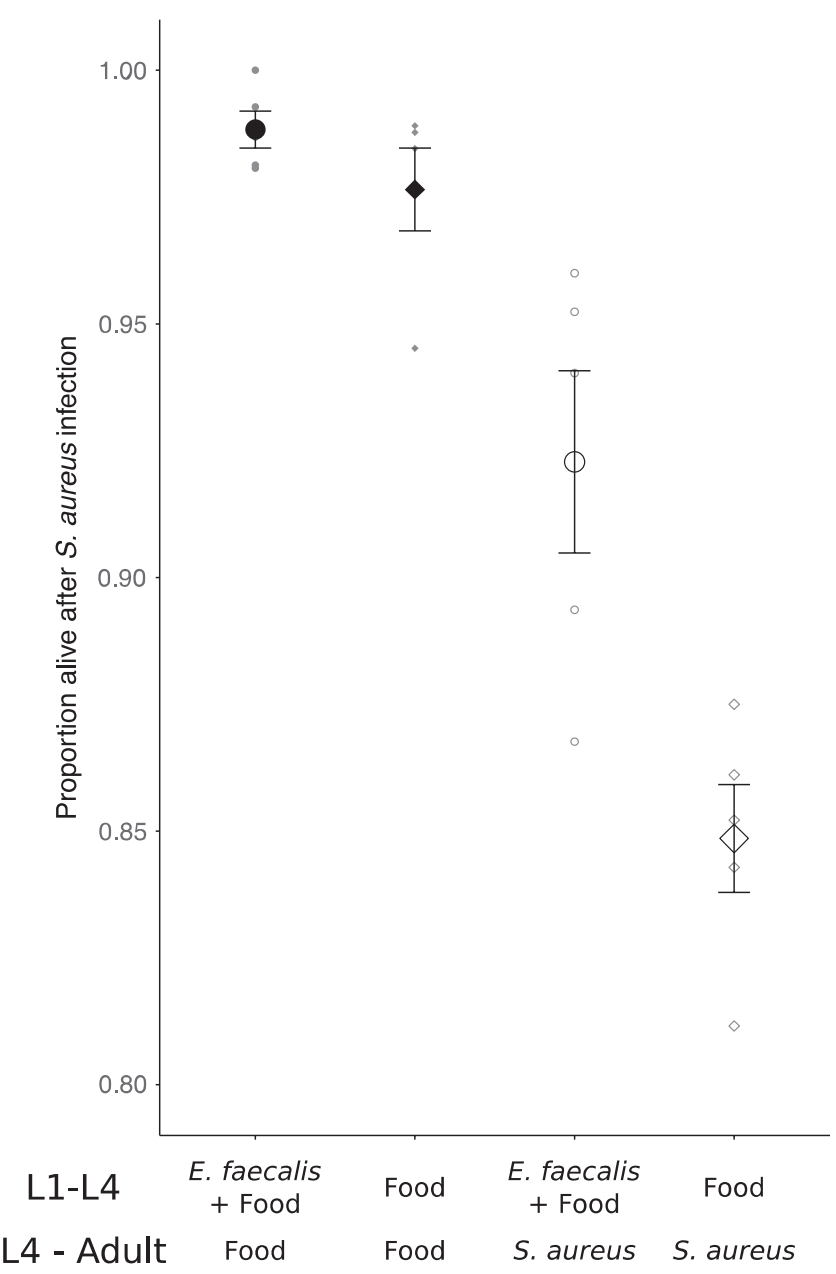

FIGURE 1 Host survival showing protective effects of Enterococcus faecalis. Early exposure of worms to E. faecalis (both ancestors) provides some degree of protection from the infection of Staphylococcus aureus. 24-hr host survival levels reveal a benefit to $E$. faecalis colonization independent of pathogen presence or absence. Circles indicate those treatment being exposed to $E$. faecalis and food in the earlier stage (L1-L4), while squares indicate food alone treatment in the earlier stage (L1-L4). Filled symbols indicate those treatments being exposed to food in the later stage, while open symbols indicate those treatments being exposed to the pathogen S. aureus in the later stage. Each symbol indicates the mean $\pm S E$ of five replicates. Axis scales were chosen to be the same across all plots

Papkou et al., 2019; Rafaluk-Mohr et al., 2018). Worms were infected with either S. aureus or exposed to food (Table 1) and left at $25^{\circ} \mathrm{C}$ for $24 \mathrm{hr}$. After this time, worms were then washed off the plates with M9 buffer once more to plate them on NGM plates seeded with food for laying eggs. Roughly, $10 \%$ of these worms was crushed and plated on $E$. faecalis selective medium (TSB $+100 \mathrm{mg} / \mathrm{ml}$ rifampicin). The remaining worms were left on food plates for $48 \mathrm{hr}$ to allow for egg laying.

To passage E. faecalis, roughly 100 E. faecalis colonies were picked and grown up shaking overnight in $600 \mu \mathrm{THB}$ at $30^{\circ} \mathrm{C}$, while worms were bleached and left to hatch overnight. This cycle was repeated for 20 experimental host generations. 
All passaged worms and $E$. faecalis samples were cryopreserved at $-80^{\circ} \mathrm{C}$. A proportion of the offspring of surviving worms were frozen in $40 \%$ DMSO, and $100 \mu \mathrm{l}$ of E. faecalis liquid culture was mixed with $100 \mu$ l of glycerol before cryopreservation.

\subsection{Host survival and fecundity assays}

All assays were conducted at the end of the evolution experiment on archived samples. Plates were randomized and fully encoded during each experiment to ensure the experimenter was blind to different treatments while collecting data.

Basic procedures were adopted from the experimental evolution, but with the following alterations to keep the assays feasible with higher accuracy when scoring dead and alive worms: 400 L1 worms were exposed to $200 \mu \mathrm{l}$ of food and E. faecalis on 6-cm NGM plates, while $60 \mu \mathrm{l}$ of $S$. aureus overnight culture was used to inoculate 6-cm TSB plates.

To assess microbe-mediated protection of different combinations of worms and E. faecalis, $400 \mathrm{~L} 1 \mathrm{~s}$ were exposed to 50:50 mixtures of E. faecalis and food for $48 \mathrm{hr}$. Worms were then washed off these plates as described above and infected with S. aureus for $24 \mathrm{hr}$ at $25^{\circ} \mathrm{C}$. Survival in form of counting dead and alive worms was then scored.

To assess any long-term fitness consequences after protective microbe exposure and pathogen infection, long-term survival and fecundity were measured. Worms were exposed as described for the survival assays. Subsequently, five females and five males were picked onto $3-\mathrm{cm}$ food seeded NGM plates at $25^{\circ} \mathrm{C}$ and then transferred to new plates every $36 \mathrm{hr}$ to avoid any confusion between offspring produced and original adults. At each time point, survival was scored. To measure fecundity, the number of worm eggs on the plates at $120 \mathrm{hr}$ since bleaching was counted.

\section{5 | Statistical analysis}

Statistical analyses were carried out with RStudio (version 1.1.463 for $\mathrm{Mac}$ ), and graphs were created with the ggplot2 package (version 2.1.0) and edited with Inkscape (version 0.91). All host survival and fecundity data were analyzed with nested binomial mixed-effects models ( $R$ package Ime4), followed by a Tukey multiple comparison tests ( $R$ package multcomp). Life span data were analyzed with Kaplan-Meier log-rank test with FDR correction for multiple testing.

\section{3 | RESULTS}

Before the start of the evolution experiment, the starting conditions were tested. Confirming previous results, E. faecalis showed some spontaneous host-protective potential against $S$. aureus. Worms (a) Sympatric pairs of coevolved worms and coevolved E. faecalis

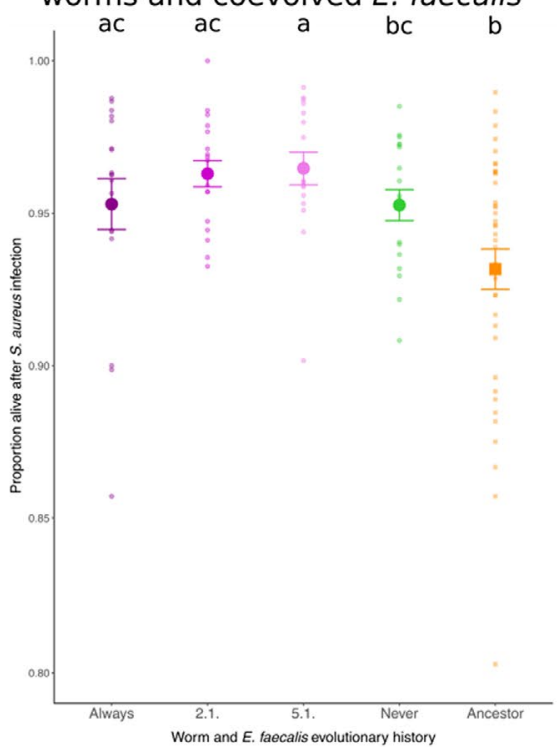

(b) Allopatric pairs of evolved worms and ancestral $E$. faecalis

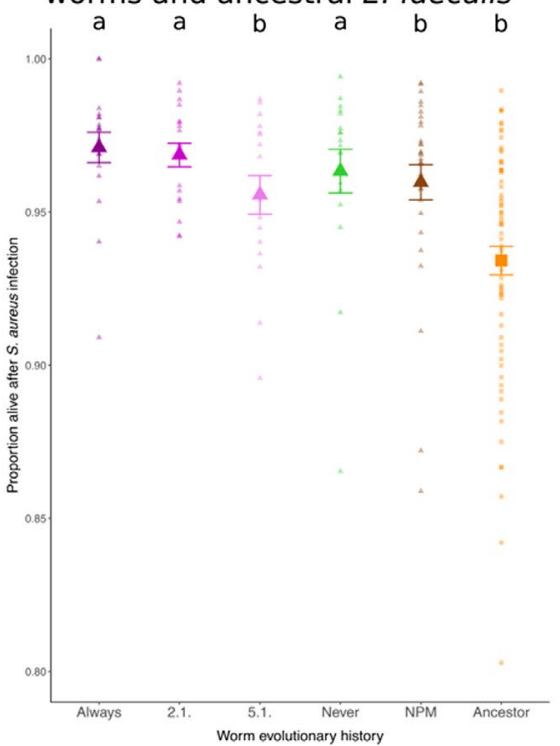

(c) Allopatric pairs of ancestral worms and evolved E. faecalis

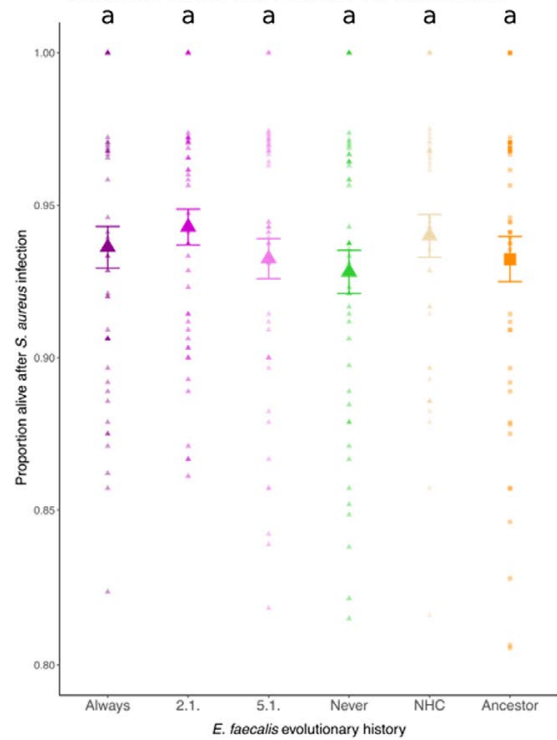

FIGURE 2 Host survival for coevolving sympatric and allopatric pairs of worms and Enterococcus faecalis. Microbe-mediated protection was assessed for (a) sympatric pairs of coevolved worms and E. faecalis, (b) allopatric pairs of evolved worms and ancestral E. faecalis, and (c) allopatric pairs of ancestral worms and evolved $E$. faecalis. Bigger symbols represent mean $\pm S E$ and consist of six biological replicates and four technical replicates. Smaller symbols indicate the data distribution. Circles indicate sympatric pairs of coevolved E. faecalis and worms, squares indicate ancestral pairs of E. faecalis and worms, and triangles indicate allopatric pairs of E. faecalis and worms. Letters indicate results of a GLMM, followed by a Tukey post hoc test. The same letter indicates no significant difference. Axis scales were chosen to be the same across all plots 
raised on E. faecalis and food survived better than those raised on food alone, independent of food or pathogen present at the later stage (general linear model, $X^{2}=10.205, d f=1, p=.001$; Figure 1). Worms infected with $S$. aureus in later life survived worse than those being exposed to food (general linear model, $X^{2}=119.643, d f=1$, $p<.001$; Figure 1). These results demonstrate the beneficial and protective effects for the host after exposure to the protective microbe E. faecalis.

Infection with S. aureus over evolutionary time in the experiment led to the substantial enhancement of microbe-mediated protection, with the evolutionary background of the sympatric pair of host and E. faecalis having a significant impact on host survival (mixed-effects model, $X^{2}=42.479, d f=4, p<.001$; Figure 2a). Higher

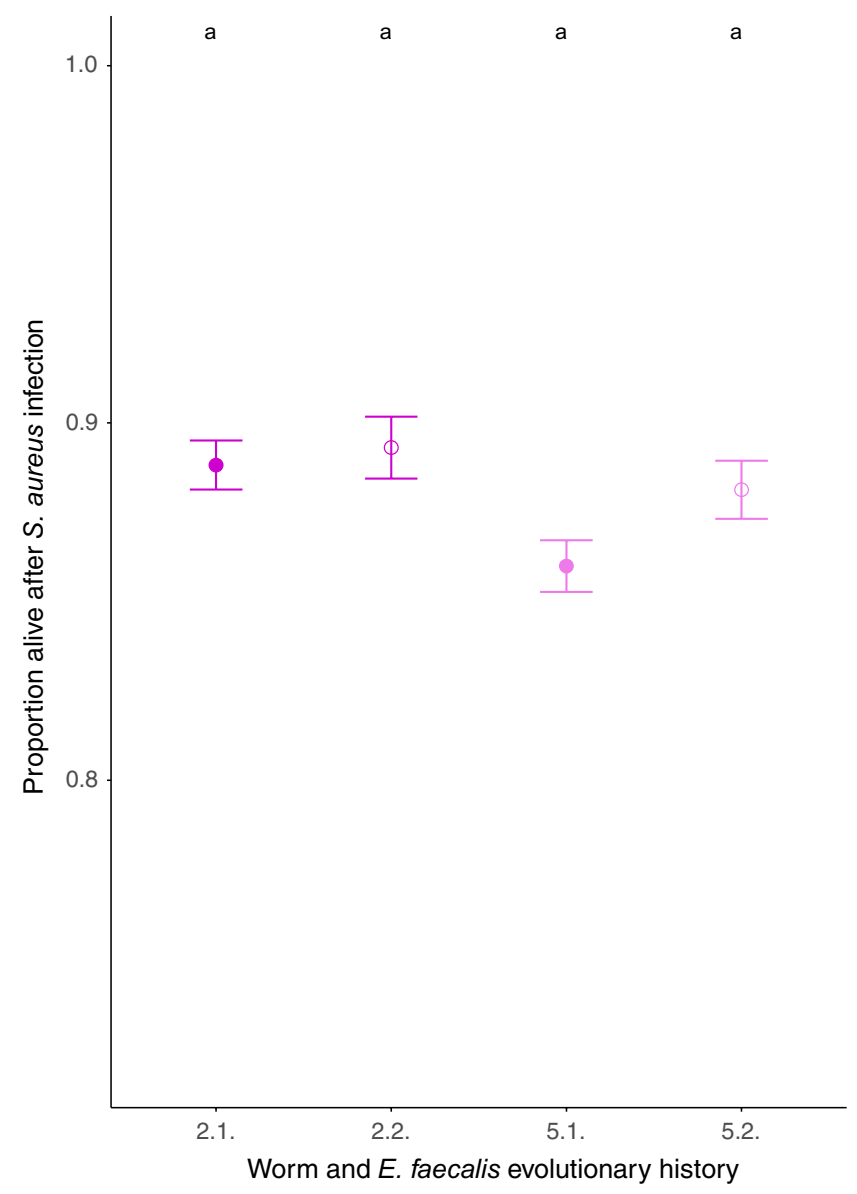

FIGURE 3 Host survival in evolutionary treatments differing in initial pathogen exposure time points. The time point of initial infection varied for infection to the pathogen every two generations (2.1. and 2.2) or every five generations (5.1. or 5.2.) but does not influence the outcome. Closed symbols indicate initial pathogen presence (host generation 1); open symbols indicate later pathogen presence (generation 2 for 2.1. and 2.2. and generation 5 for 5.1. and 5.2.). Bigger symbols represent mean $\pm S E$ and consist of six biological replicates and four technical replicates of the sympatric pairs. Smaller symbols indicate the data distribution. Letters indicate results of a GLMM, followed by a Tukey post hoc test. The same letter indicates no significant difference. Axis scales were chosen to be the same across all plots microbe-mediated protection in comparison with the Ancestor occurred in all evolutionary histories involving pathogen presence across the temporal heterogeneity treatments in our evolution experiment (always, 2.1. and 5.1.). However, this did not occur in the pathogen absence (never) treatment. Host evolutionary history alone had a significant effect on host survival (mixed-effects model, $X^{2}=35.779, d f=5, p<.001$; Figure $2 b$ ), but did not reveal the same pattern as for sympatric pairs. No effect of bacteria evolutionary history alone on infected host survival was observed (mixed-effects model, $X^{2}=3.2511, d f=5, p=.6613$; Figure 2c). Taken together, enhanced microbe-mediated protection evolved only as a product of coevolution and pathogen presence for sympatric pairs; this occurred regardless of the temporal heterogeneity.

As an additional form of pathogen heterogeneity, the impact of the timing of initial pathogen infection on the evolution of microbe-mediated protection was investigated. An effect of different initial pathogen infection time points on host survival following pathogen infection was observed (mixed-effects model: $X^{2}=7.945$, $d f=3, p=.04716$ Figure 3 ), although a Tukey post hoc test revealed no significant differences (Table S1).

Furthermore, we investigated the long-term consequences to hosts colonized by E. faecalis after $24 \mathrm{hr}$ of pathogen infection. No significant differences were observed in the long-term survival postinfection of worm hosts colonized by their sympatric E. faecalis across treatments (Kaplan-Meier log-rank test, FDR-corrected, all comparisons $p>.05$, Figure $4 a$ ). In addition, we did not find significant differences in fecundity among sympatric host- $E$. faecalis pairs (mixed-effects model, $X^{2}=3.9418$, df $=4, p=.4278$, Figure 4b).

\section{4 | DISCUSSION}

It has been shown that hosts receive the greatest benefits from protective microbes under constant pathogen infection. We hypothesized that variation in pathogen presence over time would limit the evolution of microbe-mediated protection due to the reduced benefits to the host and bacterial symbiont. In our study, enhanced pathogen defense emerged out of host-symbiont coevolutionary interactions only when pathogens were present, independent of the interval or initial presence of the pathogen. Notably, the ultimate strength of microbe-mediated protection that evolved was not impacted by the number of host generations between pathogen infections, the proportion of generations infected, or the presence of the pathogen at the first host-microbe interaction. These results suggest that resident microbes can be a form of transgenerational immunity against rare pathogen infections.

We found that microbe-mediated protection is maintained even in the prolonged absence of pathogen, but that pathogen presence is necessary for microbe-mediated protection to evolve, as previously hypothesized (Clay et al., 2005; King \& Bonsall, 2017; Lively et al., 2005). This result is unlike previous work showing that the 

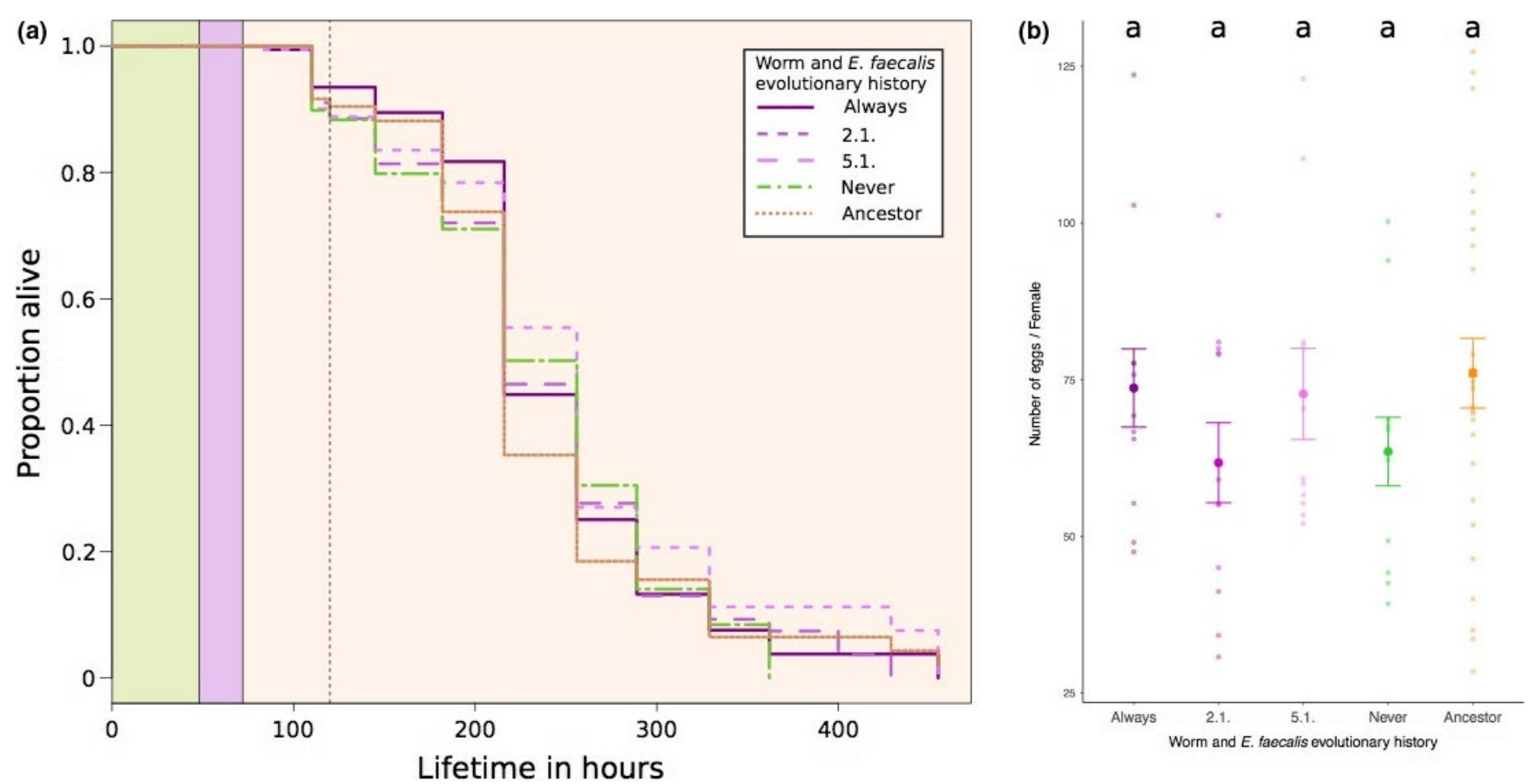

FIGURE 4 Long-term survival and fecundity of Enterococcus faecalis-colonized hosts that survived pathogen infection. (a) Long-term host survival was measured. Survival curves for sympatric pairs of worms and E. faecalis are shown as Kaplan-Meier estimates. Worms were exposed to E. faecalis and food (green), and then to Staphylococcus aureus (purple), and long-term survival was monitored on food (orange). The dotted line indicates the time point at which fecundity was measured. (b) Number of eggs/female across sympatric pairs of coevolved worms and E. faecalis. Bigger symbols represent mean $\pm S E$ and consist of six biological replicates and four technical replicates. Smaller symbols indicate the data distribution. Circles indicate sympatric pairs of coevolved E. faecalis and worms; squares indicate ancestral pairs of E. faecalis and worms. Letters indicate results of a GLMM, followed by a Tukey post hoc test. The same letter indicates no significant difference

scale of heterogeneity in abiotic conditions can affect the strength of selection for traits in some symbiotic interactions (Harrison et al., 2013). This discrepancy is potentially due to costs in our symbiotic system being ameliorated (at least in terms of host survival) in well-provisioned hosts, as hosts are provided with food alongside E. faecalis and are thus rescued from starvation (also see Dasgupta et al., 2019). Although protective symbionts can incur costs (e.g., Vorburger \& Gouskov, 2011) for their hosts, with potential for impacts on coevolutionary interactions (King \& Bonsall, 2017), it is possible that potential costs of bacterial colonization might be only detectable when hosts are stressed (Lively, 2006) or that the costs were not strong enough for us to detect (Little, Carius, Sakwinska, \& Ebert, 2002). Different measures of cost remain to be explored (e.g., life span in the complete absence of a protective microbe and a pathogen). Higher protection also does not always come with higher costs, as found in the black bean aphid-Hamiltonella defensa interaction (Cayetano, Rothacher, Simon, \& Vorburger, 2015). Thus, protective traits in an organism's commensal microbiota could be selected for under pathogen infection and easily maintained in subsequent uninfected generations.

Microbe-mediated protection was strongest between sympatric pairs when pathogens were present over evolutionary time, consistent with previous findings (Rafaluk-Mohr et al., 2018). In our study, protection emerged during coevolution after only 20 host generations, and not due to the independent evolution of either interacting species, but due to the coevolution of both species (King \& Bonsall, 2017). The time scale of these interactions is short compared to the longer shared evolutionary histories shared by other defensive mutualisms (Jousselin, Rasplus, \& Kjellberg, 2003; Quek, Davies, Itino, \& Pierce, 2004; Shoemaker et al., 2002). Nevertheless, our findings reveal the potential for microbe-mediated protection to become enhanced during the formation of a coevolving host-microbiota relationship.

In conclusion, our results show that enhanced protection in host-microbe interactions can rapidly evolve and be maintained even under infrequent pathogen infection, suggesting that resident microbes can be a form of stable, transgenerational immunity. The protective benefit of an organism's microbiota might remain undetected for several host generations until pathogens re-emerge. Future research on the failure of pathogens transmit within host populations should consider the contribution of the protective microbiota to prevent disease spread.

\section{ACKNOWLEDGMENTS}

We would like to thank the King group for help and support during the performance of the evolution experiment, especially Charlotte Rafaluk-Mohr and Maria Ordovas-Montanes. AK was supported by a fellowship from the "Studienstiftung des Deutschen Volkes." KCK 
is grateful for a Leverhulme Trust Project Grant (RPG-2015-165) and ERC Starting Grant (COEVOPRO 802242).

\section{CONFLICT OF INTEREST}

The authors declare no conflict of interest.

\section{AUTHOR CONTRIBUTION}

Anke Kloock: Conceptualization (equal); Data curation (equal); Formal analysis (lead); Investigation (equal); Visualization (lead); Writing-original draft (lead). Michael B. Bonsall: Conceptualization (supporting); Data curation (supporting); Formal analysis (supporting); Supervision (equal); Writing-review \& editing (equal). Kayla C. King: Conceptualization (equal); Data curation (supporting); Formal analysis (supporting); Funding acquisition (lead); Supervision (equal); Writing-review \& editing (lead).

\section{DATA AVAILABILITY STATEMENT}

All evolved worm and bacteria strains are cryopreserved and can be provided upon request. Raw data and all scripts that were used for statistical analysis are available via the following link: https://osf.io/ vpm9b/.

\section{ORCID}

Anke Kloock (iD https://orcid.org/0000-0002-4618-148X

Michael B. Bonsall ID https://orcid.org/0000-0003-0250-0423

Kayla C. King iD https://orcid.org/0000-0003-1393-9220

\section{REFERENCES}

Abt, M. C., \& Artis, D. (2013). The dynamic influence of commensal bacteria on the immune response to pathogens. Current Opinion in Microbiology, 16, 4-9. https://doi.org/10.1016/j.mib.2012.12.002

Altizer, S., Dobson, A., Hosseini, P., Hudson, P., \& Pascual, M. (2006). Seasonality and the dynamics of infectious diseases. Ecology Letters, 9(4), 467-484. https://doi. org/10.1111/j.1461-0248.2005.00879.x.

Barrière, A. \& Felíix, M.-A. (2005). Natural variation and population genetics of Caenorhabditis elegans, In WormBook, (Ed.), The C. elegans Research Community, WormBook, https://doi.org/10.1895/wormb ook.1.43.1, http://www.wormbook.org

Brenner, S. (1974). The genetics of Caenorhabditis elegans. Genetics, 77, 71-94.

Cabreiro, F., \& Gems, D. (2013). Worms need microbes too: Microbiota, health and aging in Caenorhabditis elegans: The C. elegans -microbe holobiont. EMBO Molecular Medicine, 5, 1300-1310. https://doi. org/10.1002/emmm.201100972

Cayetano, L., Rothacher, L., Simon, J.-C., \& Vorburger, C. (2015). Cheaper is not always worse: Strongly protective isolates of a defensive symbiont are less costly to the aphid host. Proceedings of the Royal Society B-Biological Sciences, 282, 20142333. https://doi.org/10.1098/ rspb. 2014.2333

Clay, K., Holah, J., \& Rudgers, J. A. (2005). Herbivores cause a rapid increase in hereditary symbiosis and alter plant community composition. Proceedings of the National Academy of Sciences of the United States of America, 102, 12465-12470. https://doi.org/10.1073/ pnas.0503059102

Dasgupta, M., Shashikanth, M., Bojanala, N., Gupta, A., Javed, S., \& Singh, V. (2019). Nuclear hormone receptor NHR-49 shapes immuno-metabolic response of Caenorhabditis elegans to
Enterococcus faecalis infection (preprint). Immunology. https://doi. org/10.1101/549907

Dey, S., Proulx, S. R., \& Teotónio, H. (2016). Adaptation to temporally fluctuating environments by the evolution of maternal effects. PLoS Biology, 29, e1002388.

Dillon, R. J., Vennard, C. T., \& Charnley, A. K. (2000). Exploitation of gut bacteria in the locust. Nature, 403, 851. https://doi. org/10.1038/35002669

Dong, Y., Manfredini, F., \& Dimopoulos, G. (2009). Implication of the mosquito midgut microbiota in the defense against malaria parasites. PLoS Path, 5, e1000423. https://doi.org/10.1371/journ al.ppat.1000423

Fenton, A., Johnson, K. N., Brownlie, J. C., \& Hurst, G. D. D. (2011). Solving the Wolbachia Paradox: Modeling the tripartite interaction between host, Wolbachia, and a natural enemy. The American Naturalist, 178, 333-342. https://doi.org/10.1086/661247

Finkelman, B. S. (2007). Global patterns in seasonal activity of influenza A/H3N2, A/H1N1, and B from 1997 to 2005: Viral coexistence and latitudinal gradients. PLoS One, 2(12), e1296.

Ford, S. A., Williams, D., Paterson, S., \& King, K. C. (2017). Co-evolutionary dynamics between a defensive microbe and a pathogen driven by fluctuating selection. Molecular Ecology, 26, 1778-1789. https://doi. org $/ 10.1111 / \mathrm{mec} .13906$

Friman, V.-P., \& Laakso, J. (2011). Pulsed-resource dynamics constrain the evolution of predator-prey interactions. The American Naturalist, 177, 334-345. https://doi.org/10.1086/658364

Friman, V.-P., Laakso, J., Koivu-Orava, M., \& Hiltunen, T. (2011). Pulsed-resource dynamics increase the asymmetry of antagonistic coevolution between a predatory protist and a prey bacterium: Pulsed-resource dynamics and coevolution. Journal of Evolutionary Biology, 24, 2563-2573. https://doi. org/10.1111/j.1420-9101.2011.02379.x

Garsin, D. A., Sifri, C. D., Mylonakis, E., Qin, X., Singh, K. V., Murray, B. E., ... Ausubel, F. M. (2001). A simple model host for identifying Grampositive virulence factors. Proceedings of the National Academy of Sciences of the United States of America, 98, 10892-10897. https:// doi.org/10.1073/pnas.191378698

Gravato-Nobre, M. J., \& Hodgkin, J. (2005). Caenorhabditis elegans as a model for innate immunity to pathogens. Cellular Microbiology, 7, 741-751. https://doi.org/10.1111/j.1462-5822.2005.00523.x

Gremillion-Smith, C., \& Woolf, A. (1988). Epizootiology of skunk rabies in North America. Journal of Wildlife Diseases, 24, 620-626. https://doi. org/10.7589/0090-3558-24.4.620

Harrison, E., Laine, A.-L., Hietala, M., \& Brockhurst, M. A. (2013). Rapidly fluctuating environments constrain coevolutionary arms races by impeding selective sweeps. Proceedings of the Royal Society B-Biological Sciences, 280, 20130937. https://doi.org/10.1098/rspb.2013.0937

Hiltunen, T., Friman, V.-P., Kaitala, V., Mappes, J., \& Laakso, J. (2012). Predation and resource fluctuations drive eco-evolutionary dynamics of a bacterial community. Acta Oecologica, 38, 77-83. https://doi. org/10.1016/j.actao.2011.09.010

Holden, M. T. G., Feil, E. J., Lindsay, J. A., Peacock, S. J., Day, N. P. J., Enright, M. C., ... Parkhill, J. (2004). Complete genomes of two clinical Staphylococcus aureus strains: Evidence for the rapid evolution of virulence and drug resistance. Proceedings of the National Academy of Sciences of the United States of America, 101, 9786-9791. https://doi. org/10.1073/pnas.0402521101

Hooper, L. V., Littman, D. R., \& Macpherson, A. J. (2012). Interactions between the microbiota and the immune system. Science, 336, 12681273. https://doi.org/10.1126/science.1223490

Jaenike, J., Unckless, R., Cockburn, S. N., Boelio, L. M., \& Perlman, S. J. (2010). Spread of a Drosophila defensive symbiont. Science, 329, 5.

Jansen, G., Crummenerl, L. L., Gilbert, F., Mohr, T., Pfefferkorn, R., Thänert, R., ... Schulenburg, H. (2015). Evolutionary transition from pathogenicity to commensalism: Global regulator mutations mediate 
fitness gains through virulence attenuation. Molecular Biology and Evolution, 32, 2883-2896. https://doi.org/10.1093/molbev/msv160

Jansson, H.-B. (1994). Adhesion of Conidia of Drechmeria coniospora to Caenorhabditis elegans wild type and mutants. Journal of Nematology, 26, 6 .

Jousselin, E., Rasplus, J.-Y., \& Kjellberg, F. (2003). Convergence and coevolution in a mutualism: Evidence from a molecular phylogeny of ficus. Evolution, 57, 1255. https://doi.org/10.1554/02-445

Kamada, N., Seo, S.-U., Chen, G. Y., \& Núñez, G. (2013). Role of the gut microbiota in immunity and inflammatory disease. Nature Reviews Immunology, 13, 321-335. https://doi.org/10.1038/nri3430

King, K. C. (2019). Defensive symbionts. Current Biology, 29, R78-R80. https://doi.org/10.1016/j.cub.2018.11.028

King, K. C., \& Bonsall, M. B. (2017). The evolutionary and coevolutionary consequences of defensive microbes for host-parasite interactions. BMC Evolutionary Biology, 17, 190. https://doi.org/10.1186/s1286 2-017-1030-z

King, K. C., Brockhurst, M. A., Vasieva, O., Paterson, S., Betts, A., Ford, S. A., ... Hurst, G. D. (2016). Rapid evolution of microbe-mediated protection against pathogens in a worm host. ISME Journal, 10, 19151924. https://doi.org/10.1038/ismej.2015.259

King, K. C., Delph, L. F., Jokela, J., \& Lively, C. M. (2009). The geographic mosaic of sex and the Red Queen. Current Biology, 19, 1438-1441. https://doi.org/10.1016/j.cub.2009.06.062

Koch, H., \& Schmid-Hempel, P. (2011). Socially transmitted gut microbiota protect bumble bees against an intestinal parasite. Proceedings of the National Academy of Sciences of the United States of America, 108, 19288-19292. https://doi.org/10.1073/pnas.1110474108

Kommineni, S., Bretl, D. J., Lam, V., Chakraborty, R., Hayward, M., Simpson, P., ... Salzman, N. H. (2015). Bacteriocin production augments niche competition by enterococci in the mammalian gastrointestinal tract. Nature, 526, 719-722. https://doi.org/10.1038/natur e15524

Kwiatkowski, M., \& Vorburger, C. (2012). Modeling the ecology of symbiont-mediated protection against parasites. The American Naturalist, 179, 595-605. https://doi.org/10.1086/665003

Ley, R. E., Peterson, D. A., \& Gordon, J. I. (2006). Ecological and evolutionary forces shaping microbial diversity in the human intestine. Cell, 124, 837-848. https://doi.org/10.1016/j.cell.2006.02.017

Little, T. J., Carius, H.-J., Sakwinska, O., \& Ebert, D. (2002). Competitiveness and life-history characteristics of Daphnia with respect to susceptibility to a bacterial pathogen: Cost of resistance in Daphnia. Journal of Evolutionary Biology, 15, 796-802. https://doi. org/10.1046/j.1420-9101.2002.00436.x

Lively, C. M. (2006). The ecology of virulence: Ecology of virulence. Ecology Letters, 9, 1089-1095. https://doi. org/10.1111/j.1461-0248.2006.00969.x

Lively, C. M., Clay, K., Wade, M. J., \& Fuqua, C. (2005). Competitive coexistence of vertically and horizontally transmitted parasites. Evolutionary Ecology Research, 7, 1183-1190.

London, W. P., \& Yorke, J. A. (1973). Recurrent outbreaks of measles, chickenpox, and mumps. American Journal of Epidemiology, 98(6), 453-468. https://doi.org/10.1093/oxfordjournals.aje.a121575.

Marsh, E. K., \& May, R. C. (2012). Caenorhabditis elegans, a model organism for investigating immunity. Applied and Environment Microbiology, 78, 2075-2081. https://doi.org/10.1128/AEM.07486-11

Martinez, J., Cogni, R., Cao, C., Smith, S., Illingworth, C. J. R., \& Jiggins, F. M. (2016). Addicted? Reduced host resistance in populations with defensive symbionts. Proceedings of the Royal Society B-Biological Sciences, 283, 20160778. https://doi.org/10.1098/rspb.2016.0778

Martín-Vivaldi, M., Peña, A., Peralta-Sánchez, J. M., Sánchez, L., Ananou, S., Ruiz-Rodríguez, M., \& Soler, J. J. (2010). Antimicrobial chemicals in hoopoe preen secretions are produced by symbiotic bacteria. Proceedings of the Royal Society B-Biological Sciences, 277, 123-130. https://doi.org/10.1098/rspb.2009.1377
May, G., \& Nelson, P. (2014). Defensive mutualisms: Do microbial interactions within hosts drive the evolution of defensive traits? Functional Ecology, 28, 356-363. https://doi.org/10.1111/1365-2435.12166

Maynard, C. L., Elson, C. O., Hatton, R. D., \& Weaver, C. T. (2012). Reciprocal interactions of the intestinal microbiota and immune system. Nature, 489, 231-241. https://doi.org/10.1038/nature11551

McFall-Ngai, M., Hadfield, M. G., Bosch, T. C. G., Carey, H. V., DomazetLošo, T., Douglas, A. E., ... Wernegreen, J. J. (2013). Animals in a bacterial world, a new imperative for the life sciences. Proceedings of the National Academy of Sciences of the United States of America, 110, 3229-3236. https://doi.org/10.1073/pnas.1218525110

Mendes, R., Kruijt, M., de Bruijn, I., Dekkers, E., van der Voort, M., Schneider, J. H. M., ... Raaijmakers, J. M. (2011). Deciphering the rhizosphere microbiome for disease-suppressive bacteria. Science, 332, 1097-1100. https://doi.org/10.1126/science.1203980

Moran, N. A., Tran, P., \& Gerardo, N. M. (2005). Symbiosis and insect diversification: An ancient symbiont of sap-feeding insects from the bacterial phylum Bacteroidetes. Applied and Environment Microbiology, 71, 8802-8810. https://doi.org/10.1128/AEM.71.12.8802-8810.2005

Palmer, T. M., Stanton, M. L., Young, T. P., Goheen, J. R., Pringle, R. M., \& Karban, R. (2008). Breakdown of an ant-plant mutualism follows the loss of large herbivores from an African Savanna. Science, 319, 192-195. https://doi.org/10.1126/science.1151579

Papkou, A., Guzella, T., Yang, W., Koepper, S., Pees, B., Schalkowski, R., ... Schulenburg, H. (2019). The genomic basis of Red Queen dynamics during rapid reciprocal host-pathogen coevolution. Proceedings of the National Academy of Sciences of the United States of America, 116, 923-928. https://doi.org/10.1073/pnas.1810402116

Quek, S.-P., Davies, S. J., Itino, T., \& Pierce, N. E. (2004). Codiversification in an ant-plant mutualism: Stem texture and the evolution of host use in Crematogaster (Formicidae: Myrmicinae) inhabitants of Macaranga (Euphorbiaceae). Evolution, 58, 554. https://doi.org/10.1554/03-361

Rafaluk-Mohr, C., Ashby, B., Dahan, D. A., \& King, K. C. (2018). Mutual fitness benefits arise during coevolution in a nematode-defensive microbe model. Evolution Letters, 2, 246-256. https://doi.org/10.1002/ evl3.58

Schulenburg, H., \& Ewbank, J. J. (2004). Diversity and specificity in the interaction between Caenorhabditis elegans and the pathogen Serratia marcescens. BMC Evolutionary Biology, 4, 49. https://doi. org/10.1186/1471-2148-4-49

Shoemaker, D. D., Machado, C. A., Molbo, D., Werren, J. H., Windsor, D. M., \& Herre, E. A. (2002). The distribution of Wolbachia in fig wasps: Correlations with host phylogeny, ecology and population structure. Proceedings of the Royal Society of London. Series B: Biological Sciences, 269, 2257-2267. https://doi.org/10.1098/rspb.2002.2100

Sifri, C. D., Begun, J., Ausubel, F. M., \& Calderwood, S. B. (2003). Caenorhabditis elegans as a model host for Staphylococcus aureus pathogenesis. Infection and Immunity, 71, 2208-2217. https://doi. org/10.1128/IAI.71.4.2208-2217.2003

Stiernagle, T. (2006). Maintenance of C. elegans. In WormBook, (Ed.), The C. elegans Research Community, WormBook. https://doi.org/10.1895/ wormbook.1.101.1, http://www.wormbook.org

Tan, M.-W., Mahajan-Miklos, S., \& Ausubel, F. M. (1999). Killing of Caenorhabditis elegans by Pseudomonas aeruginosa used to model mammalian bacterial pathogenesis. Proceedings of the National Academy of Sciences of the United States of America, 96, 715-720. https://doi.org/10.1073/pnas.96.2.715

Theologidis, I., Chelo, I. M., Goy, C., \& Teotónio, H. (2014). Reproductive assurance drives transitions to self-fertilization in experimental Caenorhabditis elegans. BMC Biology, 12(1), 21. https://doi. org/10.1186/s12915-014-0093-1.

Vántus, V. B., Kovács, M., \& Zsolnai, A. (2014). The rabbit caecal microbiota: Development, composition and its role in the prevention of digestive diseases - A review on recent literature in the light of molecular genetic methods. Acta Agraria Kaposváriensis, 18, 55-65. 
Vorburger, C., \& Gouskov, A. (2011). Only helpful when required: A longevity cost of harbouring defensive symbionts: Defensive symbionts reduce host longevity. Journal of Evolutionary Biology, 24, 1611-1617. https://doi.org/10.1111/j.1420-9101.2011.02292.x

\section{SUPPORTING INFORMATION}

Additional supporting information may be found online in the Supporting Information section.
How to cite this article: Kloock A, Bonsall MB, King KC.

Evolution and maintenance of microbe-mediated protection under occasional pathogen infection. Ecol Evol. 2020;10:

8634-8642. https://doi.org/10.1002/ece3.6555 